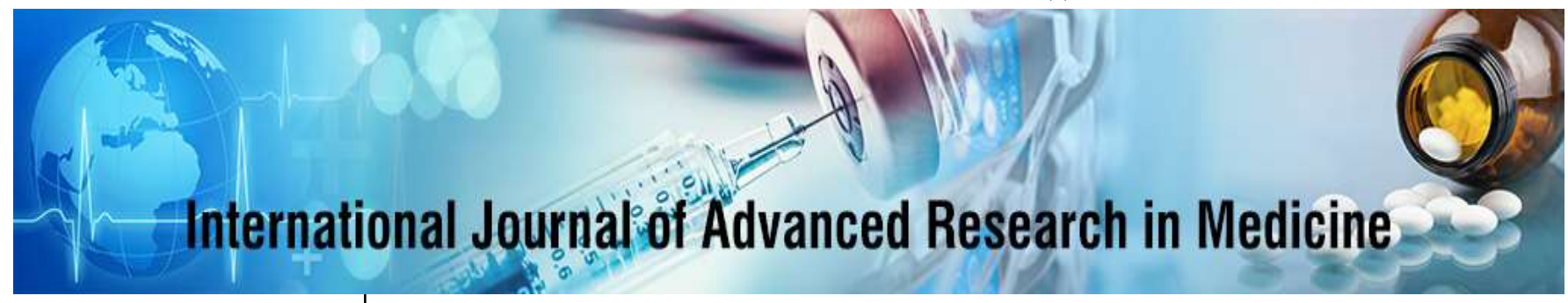

E-ISSN: 2706-9575

P-ISSN: 2706-9567

IJARM 2021; 3(1): 415-418

Received: 07-02-2021

Accepted: 25-03-2021

Dr. Soumya Ramchandra Naik Assistant Professor, Department of Psychiatry, Al- Amen Medical College, Vijayapura, Karnataka, India

Corresponding Author: Dr. Soumya Ramchandra Naik Assistant Professor, Department of Psychiatry, Al- Amen Medical College, Vijayapura, Karnataka, India

\section{Personality trait and cognitive function in subjects with bipolar disorder and comorbid alcohol intake}

\author{
Dr. Soumya Ramchandra Naik
}

DOI: https://doi.org/10.22271/27069567.2021.v3.i1g.174

\begin{abstract}
Background: Bipolar disorder is a life-threatening and complex condition leading to cognitive and personality impairment, imparting difficult short and long-term management, and affects approximately $1 \%$ of the population globally

Aims: The present trial was carried out to assess the clinical features of subjects with comorbid alcohol use with bipolar disorder and to assess the cognitive functions and personality traits in these subjects. Materials and Methods: The subjects were screened and assessed for 3 months to assess the presence of bipolar disorder as per ICD 10 criteria using SCAN and were divided into controls and cases $(n=40)$. After assessing subjects on various evaluation tools, the data were subjected to the statistical evaluation for both, case and controls, using t-test, and SPSS version 21.0, and a value of $p<0.05$ was used as the level of significance.

Results: The results showed that personality traits of openness to experience and neuroticism were seen more in subjects having bipolar disorder with comorbid use of alcohol than in subjects with Bipolar disorder only $(p<0.001)$. In subjects with Bipolar disorder and comorbid alcohol use, they took a long time to complete DSST compared to the subjects with bipolar disorder alone, and this difference was statistically significant $(p<0.001)$. FAB scores and Stroop effect scores were higher significantly in the subjects with Bipolar disorder and comorbid alcohol use $(p<0.001$ for both FAB and Stroop test).

Conclusion: The present study concludes that comorbid intake of alcohol further complicates the complex life-threatening bipolar disorder diagnosis and management. Also, in subjects with high scores of openness to experience and neuroticism, there is more probability of developing comorbid alcohol intake tendencies and related disorder development.
\end{abstract}

Keywords: Bipolar disorder, cognitive impairment, personality disorder, personality trait comorbid alcohol intake

\section{Introduction}

Bipolar disorder affects approximately $1 \%$ of the population globally and is one of the most commonly reported psychiatric disorders. Diagnosis of Bipolar disorder depends on at least 2 episodes of mood alteration and anxiety in affected subjects with complete recovery between the two episodes. The mood alteration in bipolar syndrome can be depressive or manic/hypomanic where more energy and elevated mood are noticed. As reported lately lifetime prevalence of bipolar disorder is $5 \%$ this increase is seen owing to better detection of subtype II of bipolar syndrome where depression with hypomania is seen. This condition is a life-threatening and complex condition imparting difficult short and long-term management ${ }^{[1]}$. Comorbid conditions are frequently associated with bipolar disorder causing lifetime psychiatric comorbidity in approximately $70 \%$ of affected subjects. The comorbidities further challenge the disease process and outcome. The commonly seen comorbidities with bipolar disorder are anxiety, attention deficit disorder, substance abuse, and/or personality disorders with substance use being the most common comorbidity. Alcohol intake being most important and common comorbidity may result as a symptom or self-medication. However, alcohol abuse can act as a risk factor or may cause bipolar syndrome itself ${ }^{[2]}$.

Substance abuse comorbidity in bipolar syndrome is higher than in unipolar syndrome or Schizophrenia. Co-occurrence of bipolar syndrome is seen with alcohol dependence than alcohol abuse. Alcohol use in bipolar syndrome increases the cost of treatment, length of hospital stay, the tendency to suicidal attempts, disability, and stress in subjects leading to poor prognosis, and bipolar syndrome patients respond poorly to de-addiction ${ }^{[3]}$. 
Cognitive functions are impaired in substance abuse and bipolar disorder even in respective abstinent and euthymic states. More impairment is seen associated with alcohol abuse and bipolar syndrome, especially in executive function and memory areas. Although previous literature work has shown characteristic personality traits individually with alcohol use and bipolar syndrome, very few literature works focused on comorbid substance abuse and bipolar disorder. This literature work focused on personality traits that co-occur, at least to some extent, to the combined bipolar disorder and alcohol abuse. Hence, identification of these common personality traits can help to prevent second disorder co-occurrence ${ }^{[4]}$. The present trial was carried out to assess the clinical features of subjects with comorbid alcohol use with bipolar disorder and to assess the cognitive functions and personality traits in these subjects.

\section{Materials and methods}

The present study was a cross-sectional trial carried out at Department Of Psychiatry, Al- Amen Medical College, Vijayapura, Karnataka for 3 months. The study subjects were recruited from the patients visiting the Outpatient Department. The subjects were screened and assessed for the presence of bipolar disorder as per ICD 10 criteria using SCAN (Schedules for Clinical Assessment in Neuropsychiatry). To be included in the study, the subjects had to be above 18 years of age, have both alcohol use and bipolar disorder as per ICD-10, normal vision and auditory function on evaluation, euthymic state subjects, subjects who did not take alcohol for the past 2 weeks, and subjects and their accompanier agreed to give the informed consent. Following subjects were excluded from the study: the subjects who were mentally retarded and were not in a state of giving consent, if subjects had other psychiatric illness or neurological disease, and if they had any other abuse except smoking.

The study controls were contributed by the subjects with Bipolar disorder, 18 years or more, in the euthymic state, no alcohol use history, no auditory or visual impairment on examination, and provided informed consent. The exclusion criteria for controls were mentally retarded states and were not in a state of giving consent, if subjects had other psychiatric illness or neurological disease, and if they had any other abuse except smoking. The tools used in the study were SCAN ${ }^{[5]}$, a questionnaire to record demographic characteristics and other clinic data of the subjects, Digit symbol substitution test ${ }^{[6]}$, Stroop word color test ${ }^{[7]}$, and FAB $^{[8]}$ (Frontal Assessment Battery). FAB assessment had a score of a maximum of 18 , where 12 or less was abnormal.

After final inclusion to the controls $(n=40)$ and cases $(n=40)$ group, the subjects were explained about the detailed study plans, and the consent was taken. Demographic characteristics were then recorded. The mentioned cognitive assessments were then evaluated on the given scales for all the study subjects. The collected data were subjected to the statistical evaluation for both cases and controls, using ttest, and SPSS version 21.0, and a value of $p<0.05$ was used as the level of significance.

\section{Results}

The study had a total of 80 subjects, 40 cases, and 40 controls. All 8 subjects were male. Among 40 cases, 28 subjects were alcohol dependent, and 12 subjects had alcohol abuse. An Independent t-test was applied to assess the social and demographic characteristics of the study subjects and the results are described in Table 1 . The results showed that regarding age there was no statistically significant difference $(\mathrm{p}=0.1378)$ in the two groups where controls and cases had a mean age of $34.5 \pm 8.3$ and 37.2 \pm 7.8 years respectively. Similar results were seen concerning socioeconomic status where no statistically significant difference was seen in the lower, middle, or upper socioeconomic group with $\mathrm{p}=0.06$. Also concerning employment status, $17.5 \%(\mathrm{n}=7)$ subjects in both groups were unemployed and $82.5 \% \quad(n=33)$ subjects were employed which showed no significant difference in the two groups. Similar results were seen concerning educational qualification $(\mathrm{p}=0.113)$.

The present study also evaluated the personality traits of the cases and controls of the study subjects and the results are summarized in Table 2 . The results showed that personality traits of openness to experience and neuroticism were seen more in subjects having bipolar disorder with comorbid use of alcohol than in subjects with Bipolar disorder only. The openness to experience had scores of $30.52 \pm 3.606$ and $34.88 \pm 3.572$ in the control and case groups with $p<0.001$ depicting statistical significance. Similarly, Neuroticism had respective scores of $22.70 \pm 4.380$ and $34.08 \pm 6.527$ in controls and cases which showed significant difference statistically ( $p<0.001)$. Concerning extraversion, both groups had low scores $(\mathrm{p}=0.336)$. For conscientiousness and agreeableness as personality traits, average scores were seen ( $\mathrm{p}=0.613$ and 0.719 respectively).

Concerning cognitive variables assessed in the present study including the Digit symbol substitution test, Stroop word color test, and FAB (Frontal Assessment Battery), the results are described in Table 3. It was seen that subjects with Bipolar disorder and comorbid alcohol use, took a long time to complete DSST compared to the subjects with bipolar disorder alone, and this difference was statistically significant $(p<0.001)$. FAB scores and Stroop effect scores were higher significantly in the subjects with Bipolar disorder and comorbid alcohol use with respective values of $29.28 \pm 3.198$ and $29.02 \pm 3.243$ respectively for FAB and $30.52 \pm 3.606$ and 31.36 \pm 3.222 for Stroop test $(p<0.001$ for both FAB and Stroop test).

\section{Discussion}

The socio-demographic study data showed that regarding age there was no statistically significant difference $(\mathrm{p}=0.1378)$ in the two groups where controls and cases had a mean age of $34.5 \pm 8.3$ and $37.2 \pm 7.8$ years respectively. Similar results were seen concerning socioeconomic status where no statistically significant difference was seen in the lower, middle, or upper socioeconomic group with $\mathrm{p}=0.06$. Also concerning employment status, $17.5 \%(n=7)$ subjects in both groups were unemployed and $82.5 \%(n=33)$ subjects were employed which showed no significant difference in the two groups. Similar results were seen concerning educational qualification $(\mathrm{p}=0.113)$. These findings were in agreement with the findings of Thompson JM et al. ${ }^{[9]}$ in 2005 where similar results concerning demographic data were seen in alcohol comorbid subjects with bipolar disorder.

The present study showed that personality traits of openness to experience and neuroticism were seen more in subjects having bipolar disorder with comorbid use of alcohol than in 
subjects with Bipolar disorder only. The openness to experience had scores of $30.52 \pm 3.606$ and $34.88 \pm 3.572$ in the control and case groups with $p<0.001$ depicting statistical significance. Similarly, Neuroticism had respective scores of $22.70 \pm 4.380$ and $34.08 \pm 6.527$ in controls and cases which showed significant difference statistically $(p<0.001)$. Concerning extraversion, both groups had low scores $(\mathrm{p}=0.336)$. For conscientiousness and agreeableness as personality traits, average scores were seen ( $\mathrm{p}=0.613$ and 0.719 respectively). These findings were consistent with the previous work of Harley JA et al. ${ }^{[10]}$ in 2011 and Haro G et al. ${ }^{[11]}$ in 2007 where high neuroticism scores were seen in bipolar disorder as well as bipolar disorder with comorbid alcohol intake.

Concerning cognitive variables assessed in the present study including Digit symbol substitution test, Stroop word color test, and FAB (Frontal Assessment Battery), it was seen that subjects with Bipolar disorder and comorbid alcohol use, took longer time to complete DSST compared to the subjects with bipolar disorder alone, and this difference was statistically significant $(p<0.001)$. FAB scores and Stroop effect scores were higher significantly in the subjects with Bipolar disorder and comorbid alcohol use with respective values of $29.28 \pm 3.198$ and $29.02 \pm 3.243$ for $F A B$ and $30.52 \pm 3.606$ and 31.36 \pm 3.222 respectively for Stroop test ( $p$ $<0.001$ for both FAB and Stroop test). These results also were similar to the findings of Levy B et al. ${ }^{[12]}$ in 2008 and Marshall DF et al. ${ }^{[13]}$ in 2012 where low FAB scores were seen in comorbid alcohol with bipolar disorder than bipolar disorder alone.

Table 1: Demographic characteristics of the study subjects

\begin{tabular}{|c|c|c|c|c|c|}
\hline Parameter & \multicolumn{2}{|r|}{ Group } & Mean \pm S.D & t-value & p-value \\
\hline \multicolumn{6}{|l|}{ Age } \\
\hline & \multirow{2}{*}{\multicolumn{2}{|c|}{$\begin{array}{c}\text { Control (Bipolar) } \\
\text { Test (Bipolar with alcohol) }\end{array}$}} & $34.5 \pm 8.3$ & \multirow{2}{*}{1.499} & \multirow{2}{*}{0.1378} \\
\hline & & & $37.2 \pm 7.8$ & & \\
\hline Socio-economic status & Control (Bipolar) & Test (Bipolar with alcohol) & & & \\
\hline Lower & B $30(75 \%)$ & $26(65 \%)$ & & & \\
\hline Middle & $2(5 \%)$ & $7(17.5 \%)$ & & & 0.06 \\
\hline Upper & $8(20 \%)$ & $7(17.5 \%)$ & & & \\
\hline \multicolumn{6}{|l|}{ Employment state } \\
\hline Unemployed & $7(17.5 \%)$ & $7(17.5 \%)$ & & & \multirow[b]{2}{*}{ - } \\
\hline Employed & $33(82.5 \%)$ & $33(82.5 \%)$ & & & \\
\hline \multicolumn{6}{|l|}{ Educational qualification } \\
\hline Uneducated & $20(50 \%)$ & $24(60 \%)$ & & & \multirow{4}{*}{0.113} \\
\hline Undergraduate & $6(15 \%)$ & $9(22.5 \%)$ & & & \\
\hline Graduate & $12(30 \%)$ & $7(17.5 \%)$ & & & \\
\hline Post-graduate & $2(5 \%)$ & $0(-)$ & & & \\
\hline
\end{tabular}

Table 2: Personality traits in the Study Groups (Cases and Controls)

\begin{tabular}{|c|c|c|c|c|}
\hline \multirow{2}{*}{ Parameter } & \multicolumn{2}{|c|}{ Mean \pm S.D } & \multirow{2}{*}{ t-value } & \multirow{2}{*}{ p-value } \\
\cline { 2 - 5 } & Control (Bipolar, $\mathrm{n}=40$ ) & Test (Bipolar with alcohol, $\mathrm{n}=40$ ) & & \\
\hline Conscientiousness & $31.74 \pm 3.474$ & $31.36 \pm 3.222$ & 0.507 & 0.613 \\
\hline Openness to experience & $30.52 \pm 3.606$ & $34.88 \pm 3.572$ & 5.432 & $<0.001$ \\
\hline Agreeableness & $29.28 \pm 3.198$ & $29.02 \pm 3.243$ & 0.361 & 0.719 \\
\hline Extraversion & $22.28 \pm 2.615$ & $21.70 \pm 2.742$ & 0.968 & 0.336 \\
\hline Neuroticism & $22.70 \pm 4.380$ & $34.08 \pm 6.527$ & 9.156 & $<0.001$ \\
\hline
\end{tabular}

Table 3: Cognitive Variables in the Study Groups (Cases and Controls)

\begin{tabular}{|c|c|c|c|c|}
\hline \multirow{2}{*}{ Parameter } & \multicolumn{2}{|c|}{ Mean \pm S.D } & \multirow{2}{*}{ t-value } & \multirow{2}{*}{ p-value } \\
\cline { 2 - 5 } & Control (Bipolar, n=40) & Test (Bipolar with alcohol, $\mathbf{n}=\mathbf{4 0}$ ) & & \\
\hline Digit symbol substitution test (DSST) & $31.74 \pm 3.474$ & $31.36 \pm 3.222$ & 0.507 & $<0.001$ \\
\hline Stroop Test & $30.52 \pm 3.606$ & $34.88 \pm 3.572$ & 5.432 & $<0.001$ \\
\hline FAB Score (Total) & $29.28 \pm 3.198$ & $29.02 \pm 3.243$ & 0.361 & $<0.001$ \\
\hline
\end{tabular}

\section{Conclusion}

Within its limitations, the present study concludes that comorbid intake of alcohol further complicates the complex life-threatening bipolar disorder diagnosis and management. Also, in subjects with high scores of openness to experience and neuroticism, there is more probability of developing comorbid alcohol intake tendencies and related disorder development. However, the study had few limitations including a small sample size, short monitoring period, geographical area biases, no consideration of different drugs by different study subjects, cross-sectional nature of the study, and non-consideration of other substance use and abuse. Hence, more longitudinal studies with larger sample size and longer monitoring periods are required to reach a definitive conclusion.

\section{References}

1. McIntyre R, Calabrese JR. Bipolar depression: the clinical characteristics and unmet needs of a complex disorder. Curr Med Res Opin 2019;35:1993-2005.

2. Katzman MA, Bilkey TS, Chokka PR, Fallu A, Klassen LJ. Adult ADHD and comorbid disorders: clinical implications of a dimensional approach. BMC Psychiatry 2017;17:302.

3. Pompili M, Serafini G, Innamorati M, Dominici G, Ferracuti S, Kotzalidis GD, et al. Suicidal behavior and 
alcohol abuse. Int $\mathrm{J}$ Environ Res Public Health 2010;7:1392-1431.

4. Balanzá-Martínez V, Crespo-Facorro B, GonzálezPinto A, Vieta E. Bipolar disorder comorbid with alcohol use disorder: focus on neurocognitive correlates. Front Physiol 2015;6:108.

5. Wing JK, Babor T, Brugha T, Burke J, Cooper JE, Giel $\mathrm{R}$, et al. SCAN. Schedules for Clinical Assessment in Neuropsychiatry. Arch Gen Psychiatry 1990;47:589-93.

6. Joy S, Kaplan E, Fein D. Speed and memory in the WAIS-III Digit Symbol-Coding subtest across the adult lifespan. Arch Clin Neuropsychol 2004;19:759-67.

7. Alexander M, Benson D, Stuss D. Frontal lobes and language. Brain and Language 1989;37:656-91.

8. The Frontal Assessment Battery (FAB): normative data from an Italian sample and performances of patients with Alzheimer's disease and frontotemporal dementia. Alessandro Iavaronea Functional Neurology 2004;19:191-5.

9. Thompson JM, Gallagher P, Hughes JH, Watson S, Gray JM, Ferrier IN, et al. Neurocognitive impairment in euthymic patients with bipolar disorder. Br. J. Psychiatry 2005;186:32-40.

10. Harley JA, Wells JE, Frampton MA, Joyce PR. Bipolar disorder and the TCI: higher self-transcendence in bipolar disorder compared to major depression, Depression Research and Treatment 2011, 529638.

11. Haro G, Calabrese JR, Larsson C, Shirley ER, Martín $\mathrm{E}$, Leal C, et al. The relationship of personality traits to substance abuse in patients with bipolar disorder. Eur Psychiatry 2007;22:305-8.

12. Levy B, Benedetta A. Monzani, Matthew R. Stephansky, Roger D. Weiss. Neurocognitive impairment in patients with co-occurring bipolar disorder and alcohol dependence upon discharge from inpatient care. Psychiatry Res 2008;161:28-35.

13. David Marshall F, Sara Walker J. Greater executive and visual memory dysfunction in comorbid bipolar disorder and substance use disorder. Psychiatry Res 2012;200:252-7. 